\section{Seroprevalencia de la infección por Trypanosoma cruzi y factores asociados en un área endémica de Venezuela}

\author{
Seroprevalence for Trypanosoma cruzi infection and \\ associated factors in an endemic area of Venezuela
}

\author{
${ }^{1}$ Decanato de Ciencias \\ de la Salud, Universidad \\ Centroccidental Lisandro \\ Alvarado, Barquisimeto, \\ Venezuela. \\ 2 Instituto Nacional de \\ Investigaciones Agrícolas, \\ Barquisimeto, Venezuela. \\ ${ }^{3}$ Decanato de Ciencias \\ de la Salud, Universidad \\ Centroccidental Lisandro \\ Alvarado, Barquisimeto, \\ Venezuela. \\ 4 Decanato de Ciencias \\ Veterinarias, Universidad \\ Centroccidental Lisandro \\ Alvarado, Barquisimeto, \\ Venezuela. \\ 5 Facultad de Ciencias, \\ Universidad de Los Andes, \\ Mérida, Venezuela. \\ Correspondence \\ R. Bonfante-Cabarcas \\ Decanato de Ciencias de \\ la Salud, Universidad \\ Centroccidental Lisandro \\ Alvarado. \\ Av. Libertador con Andrés \\ Bello, Barquisimeto, Lara - \\ 3001, Venezuela. \\ rcabarca@ucla.edu.ve
}

\begin{abstract}
This study investigated risk factors associated with positive serological status for Trypanosoma cruzi antibodies in 26 rural communities including 905 households, 2,156 humans, and 333 dogs in Lara State, Venezuela. Serology was performed with ELISA and MABA. Data were obtained from entomological, demographic, and clinical surveys. Risk factors were determined through binary logistic regression. Seroprevalence was $7.24 \%$ in humans and $6.9 \%$ in canines. Positive serological status was positively associated with the Rhodnius prolixus vector, age, maternal history of Chagas disease, tobacco chewing, presence of mammals and birds in the household, household disarray, mud-and-wattle outbuildings, and animal nests and burrows in the peridomicile, and negatively associated with tobacco and alcohol consumption, history of cancer, and storage deposits in the peridomile. In conclusion, Chagas disease in this rural area is an old phenomenon transmitted by $R$. prolixus or by the transplacental route, associated with socio-cultural habits related to poverty, sylvatic surroundings, and the host's medical history.
\end{abstract}

Chagas Disease; Risk Factors; Serology
Rafael Bonfante-Cabarcas 1 Claudina Rodríguez-Bonfante 1 Belkys Oviol Vielma 1,2 Douglas García 3 Alexander Mogollón Saldivia 4 Elis Aldana 5 Juan Luis Concepción Curvelo 5

\section{Introducción}

Actualmente se considera que la enfermedad de Chagas es localmente transmitida en 19 países del continente americano, existiendo entre 8 y 15 millones de individuos infectados, con tasas de prevalencia de un $1,45 \%$. Se estima que cada año aparecen 41.200 casos nuevos, con una morbilidad de 1.772.365 individuos y una mortalidad de 12.500 personas. La población en riesgo ha sido reportada entre 25 y 109 millones de personas en el continente americano 1,2,3,4.

En Venezuela, en los últimos cuarenta años se ha observado un descenso progresivo de los índices de la prevalencia de la infección en humanos, desde un 44,5\% en la década de los años 50 a 60, hasta un 8,3\% en el año 2000 5. Estos resultados han sido producto de la puesta en práctica de acciones que incluyen: sustitución de viviendas, control de vectores, cribado de sangre y programas de prevención en áreas vulnerables 6 . Estas medidas han reducido la incidencia anual de 10 por cada 1.000 habitantes en 1950 a 1 por cada 1.000 habitantes en $1980^{5}$. No obstante, recientemente en la región central de Venezuela han sido reportados brotes epidémicos por contaminación oral, asociados a la presencia de Panstrongylus geniculatus en las fuentes alimentarias 7.

Pocos estudios han sido realizados en Venezuela sobre el conocimiento de los factores de riesgo asociados a la enfermedad de Chagas. 
En un estudio realizado en aéreas rurales del estado Barinas, principalmente infestado por Rhodnius prolixus, se obtuvo -como factores de riesgo asociados a una infestación triatomínica del $8 \%$ - el número de residentes en la vivienda, la presencia de "troja" en el peridomicilio y la falta de rociamiento con insecticidas en los últimos 12 meses. Los factores de riesgo asociados a una seropositividad del 3,3\% fueron la edad y la presencia de suelos de tierra en las viviendas, mientras que se encontraron como factor de protección la presencia de palmas en un radio de 31 a 60 metros de la vivienda 8 .

En el estado Lara (parroquia Xaguas), a diferencia del estado Barinas, el vector predominante fue Triatoma maculata, quien mostró capacidad de infestación (índice de infestación de 16,4\%) y colonización (39,1\% índice de colonización) de viviendas humanas. La presencia de T. maculata en el domicilio y sus alrededores fue asociado con la presencia de gallinas, desorden en el entorno, cabras, aves de corral y/o distribución del domicilio ${ }^{9}$.

A pesar de los logros obtenidos en el programa de control de la enfermedad de Chagas, la transmisión de la enfermedad de Chagas en Venezuela no ha sido interrumpida, por el contrario, recientes reportes parecen indicar que existe un incremento de la transmisión en los últimos años en las zonas endémicas donde ha sido aplicado el programa, a pesar de que los índices vectoriales han disminuido significativamente 8,10 .

Por lo tanto, no comprendemos totalmente cómo ocurre actualmente la transmisión, incluso después de 40 años de control, lo cual indica que se necesitan estudios que correlacionen la infección humana con datos personales, demográficos, ambientales y entomológicos para aportar nuevas evidencias que ayuden a comprender el problema y a establecer medidas educativas de control de la enfermedad.

En el presente trabajo realizamos un estudio seroepidemilógico y entomológico en la parroquia San Miguel localizada en el municipio Urdaneta en el estado Lara con el objetivo de evaluar la prevalencia de la infección y su asociación con factores demográficos, entomológicos, ecológicos, socioculturales y antecedentes médicos personales o familiares.

\section{Materiales y métodos}

\section{Área de estudio}

El estudio se realizó en la parroquia San Miguel, del municipio Urdaneta, estado Lara, Venezuela, ubicada a $10^{\circ} 35^{\prime} 35^{\prime \prime}$ latitud norte y $69^{\circ} 27^{\prime} 56^{\prime \prime}$ longitud oeste. La mayor parte del territorio se encuentra en un gradiente altitudinal entre 500 y 1.000 metros sobre el nivel del mar, con un clima continental estacional, temperaturas entre $22^{\circ} \mathrm{C}$ y $25^{\circ} \mathrm{C}$, con suelos kastanozems, pluviosidad entre 500 y $800 \mathrm{~mm}$ anuales, una vegetación predominantemente compuesta de matorrales y espinares. Las actividades humanas predominantes en la zona son la agricultura (sisal, zábila y judía) y la cría de caprinos.

La parroquia cuenta con una población de 12.026 habitantes y 3.304 viviendas, 26 comunidades rurales fueron escogidas intencionalmente para el estudio, por estar situadas en el área de influencia de los ambulatorios de salud de Aguada Grande, Los Planes, Cuerilles, La San Miguel, San Miguel y San Pedro de Monserrat, y de esta manera integrar el trabajo científico al trabajo comunitario de prevención para la salud.

\section{Estudio epidemiológico}

Se trata de un estudio prospectivo transversal, donde la unidad de muestreo fueron las viviendas, seleccionadas por conglomerados no estratificados a partir de mapas actualizados de las comunidades. Inicialmente, se asumió un nivel de confianza del 99\%, un error máximo admisible de 1 ,65 y una prevalencia previa del $5 \%$. La muestra quedó representada por 905 viviendas. Los criterios de inclusión de los habitantes de las viviendas seleccionadas fueron: residentes permanentes de la vivienda, sin discriminación de sexo o edad (incluyendo los niños lactantes menores a 12 meses), que aceptaran de manera voluntaria, con consentimiento informado firmado por el individuo o por sus representantes. Se incluyeron los cánidos que fueran lo suficientemente dóciles para permitir los procedimientos experimentales bajo consentimiento de los dueños. En cada vivienda se aplicó una encuesta epidemiológica para la obtención de datos demográficos, identificación de factores de riesgo y conocimientos sobre la enfermedad de Chagas.

La recolección de los datos seroepidemiológicos fue realizado entre enero del 2005 a diciembre del 2008. Cada vivienda fue visitada y evaluada por un equipo conformado por un coordinador médico o sociólogo, dos a cuatro estudiantes de Medicina del sexto año de carrera, un médico veterinario o estudiante de Medicina Veterinaria y dos miembros del Comité de Salud.

En total se evaluaron 2.156 personas, 1.092 (50,65\%) del género masculino (obreros de la agricultura y estudiantes); 1.064 (49,34\%) del género femenino (oficios del hogar, estudiantes y obreros de la agricultura). La distribución por edad de la muestra fue la siguiente: 0-10 años 
el 23.54\%, 11-20: 23,32\%, 21-40: 26,58\%, 41-60: $17,49 \%$ y > 60 años $11,08 \%$. El 2,73\% era analfabeto, $16,1 \%$ sabía leer, pero no tenía educación formal, 47,79\% tenía un nivel primario, 30,24\% un nivel secundario y $3,15 \%$ universitario. El $50 \%$ de las familias estaban en el estrato marginal o $\mathrm{V}, 33,57 \%$ en el estrato obrero o IV, $12,86 \%$ en el estrato medio bajo o III, 3,57\% en el estrato medio alto o II y ninguna familia estaba en el estrato alto o I.

Un total de 182 (7,79\%; 99 del sexo masculino y 100 entre los 21 a los 40 años) no fueron evaluados por ausencias sucesivas, por imposibilidad técnica para la toma de la muestra por venopunción o por negación voluntaria.

\section{Diagnóstico serológico y parasitológico}

Por venopunción se extrajeron de 5 a $7 \mathrm{ml}$ de sangre a cada individuo humano o canino, a partir de la cual se obtuvo el suero por centrifugación, el cual fue fraccionado por duplicado, transportado entre $2-5^{\circ} \mathrm{C}$ y almacenado a $-70^{\circ} \mathrm{C}$ hasta su uso.

La determinación de anticuerpos anti-Trypanosoma cruzi en el suero fue realizada mediante las técnicas de ELISA (Enzyme-Linked Immunoabsorbent Assay) y MABA (Multiple Antigen Blot Assay; ensayo de inmunoblot de tres bandas), utilizando los antígenos recombinantes PGR31-His, PGR30-His y PGR24-His de T. cruzi y anticuerpos secundarios tipo anti-IgG humana o canina específicas para la cadena $\gamma$, conjugada a la enzima peroxidasa. El MABA fue considerado positivo cuando al menos dos de las tres bandas de los antígenos recombinantes fueron visibles. Las muestras se consideraron positivas sólo si ambas pruebas ELISA y MABA resultasen reactivas y negativa si una o ambas pruebas fuesen no reactivas 9 .

\section{Estudio entomológico}

La recolección de triatominos se realizó a través de: (a) la búsqueda pasiva realizada por los habitantes de las comunidades, a quienes se les entregó envases y se entrenaron para recolectar los especímenes en la vivienda o en el peridomicilio; (b) la revisión sistemática de la vivienda y peridomicilio, por dos miembros del equipo de investigación, por espacio de una hora, utilizando pinzas largas, linternas e insecticidas como excitantes. Los triatominos capturados se clasificaron según clave de Lent \& Wygodzinsky 11. El contenido de la porción distal del intestino del insecto se diluyó en solución salina $0.9 \%$ y la presencia o ausencia de T. cruzi fue determinada por microscopía óptica de acuerdo a sus caracte- rísticas morfológicas. Los siguientes índices vectoriales fueron determinados: infestación, colonización, coinfestación, infección y dispersión, en concordancia con criterios establecidos por la Organización Panamericana de la Salud (OPS) en el año 200312.

\section{Análisis de los datos}

Los datos son presentados en valores absolutos, porcentuales e índices numéricos. Con el fin de evaluar las relaciones existentes entre factores de riesgo epidemiológico con los individuos seropositivos o con las casas donde habita al menos un individuo seropositivo, se realizó un análisis de regresión logística binaria, utilizando el programa SPSS, versión 11.0.1 (SPSS Inc., Chicago, EE.UU.). Con excepción de la edad, los factores independientes se categorizaron como variables dicotómicas (ausencia o presencia del factor). Un odds ratio (OR) con un intervalo de 95\% de confianza (IC95\%) y un nivel de significancia de 0,05 se utilizó en el análisis multivariado para determinar el efecto independiente de las asociaciones. Las variables no significativas fueron eliminadas por eliminación progresiva paso a paso.

Dado que la seropositividad estuvo asociada con la edad (Figura 1), se decidió hacer un estudio de casos y controles tomando todos los individuos mayores de 55 años de ambos grupos serológicos, mientras que los individuos menores de 55 años fueron pareados con individuos seronegativos por edad, comunidad de procedencia y sexo. Para incrementar la población de seropositivos se tomaron casos y controles pareados de un estudio previo realizado en la parroquia Xaguas 9 . En total se incluyeron 392 seronegativos y 167 seropositivos.

Para el análisis, las variables independientes fueron agrupadas en factores relacionados: (1) vía de transmisión de la enfermedad de Chagas (conocimiento del vector; contacto lesivo con el vector; antecedentes de vivir en una comunidad infestada con vectores; antecedentes de vivir en una comunidad infestada por vectores, donde experimentó un contacto lesivo con el vector; antecedentes de haber recibido transfusiones de sangre; antecedentes de comer carne de animales silvestres y madre con antecedentes de enfermedad de Chagas); (2) hábitos biológicos y antecedentes personales patológicos de los individuos (fumar cigarrillo o tabaco, masticación de la pasta de tabaco denominada chimó o consumo de alcohol; antecedentes de alergias, diabetes, hipertensión arterial, enfermedades autoinmunes, infecciones bacterianas que merecieron hospitalización, helmintiasis y antecedentes de cáncer personal o familiar); (3) aves que viven o 
1a) Logaritmo de los valores absolutos de los individuos seronegativos y seropositvos, respectivamente, en relación con los rangos de edad.

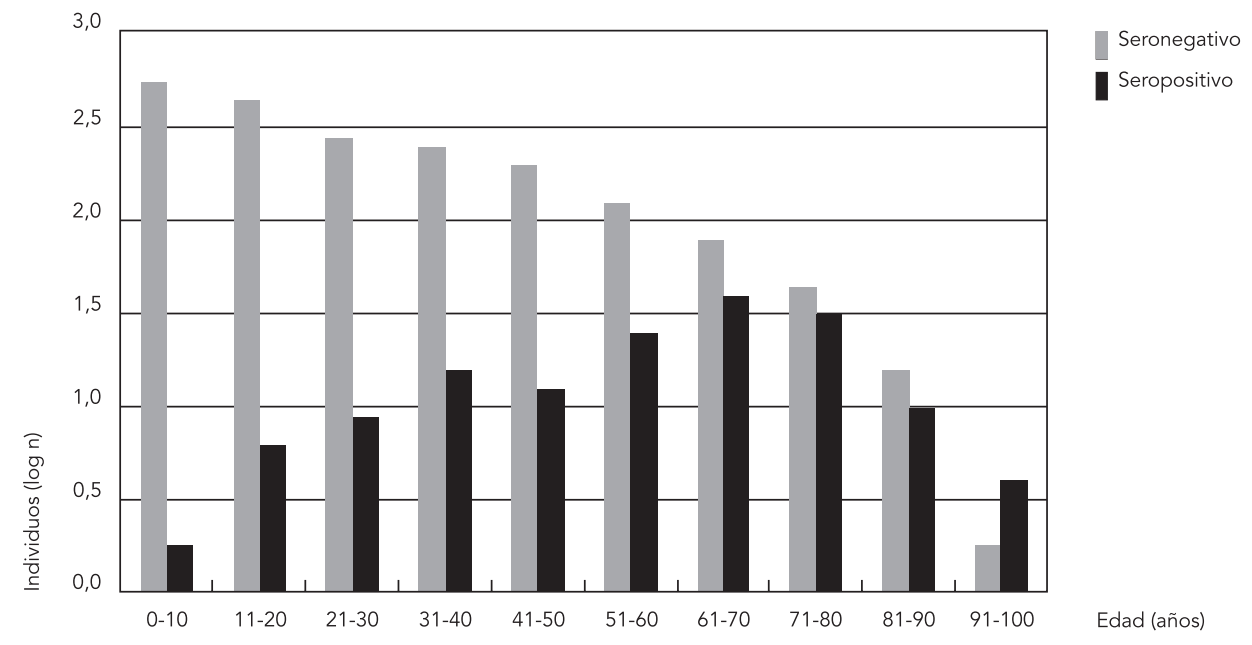

1b) Datos de prevalencia en relación con la edad.

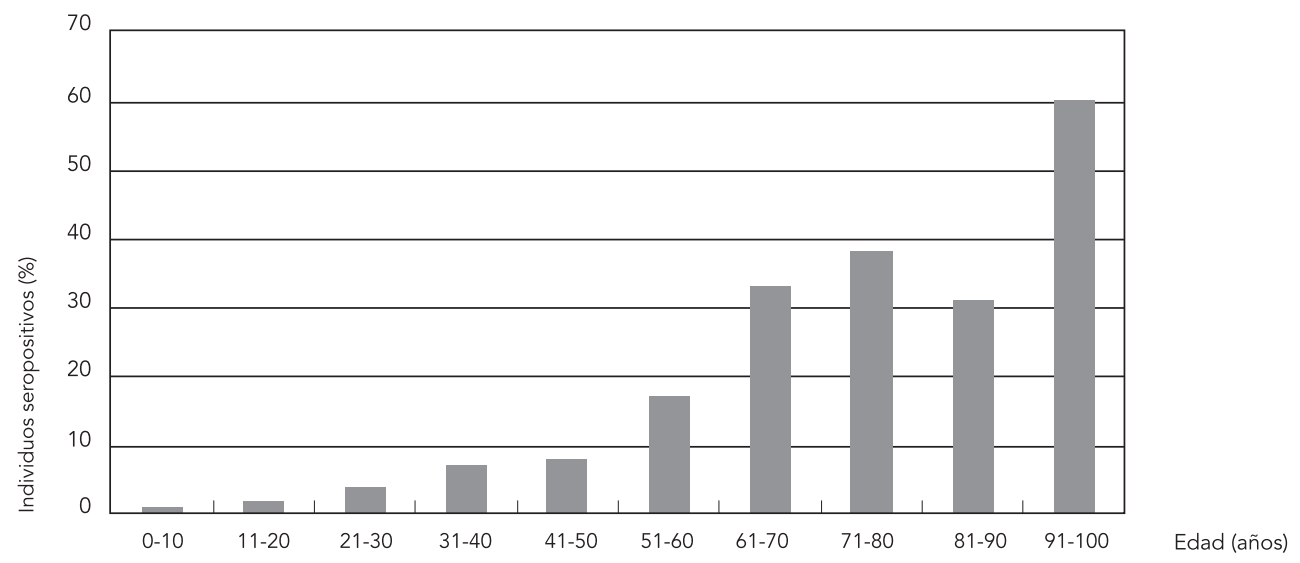

frecuentan la vivienda (gallinas, pavos, gansos, pájaros y palomas); (4) mamíferos que viven o frecuentan la vivienda (perros, gatos, ovinos, caprinos, porcinos, bovinos, roedores, zarigüeyas (Didelphys marsupialis), armadillos (Dasypus novemcinctus), perezosos (Bradipus variegatus), osos hormigueros (Tamandua sp.) y murciélagos (Chiroptera sp.); (5) distribución y orden del domicilio y peridomicilio (objetos, mobiliario y/o ropa mal arreglados; artefactos viejos, dañados y/o desechos acumulados; materiales de cons- trucción en el domicilio; limpieza inadecuada del domicilio, basura libre en el domicilio); (6) distribución y orden del peridomicilio (desechos de vidrio, plástico o metal; recipientes vacíos y/o cauchos; basura libre; artefactos domésticos o laborales no funcionales acumulados y limpieza inadecuada); y (7) construcciones anexas o hábitats en el peridomicilio (depósitos de herramientas de trabajo y utensilios del hogar; anexos de bajareque; gallineros; palomares; nidos de pájaros y cuevas de animales silvestres). 


\section{Consideraciones éticas}

El protocolo del estudio fue revisado y aprobado por el Comité de Ética del Decanato de Ciencias de la Salud Dr. Pablo Acosta Ortiz.

\section{Resultados}

\section{Seroprevalencia}

De los 2.156 individuos estudiados, se encontraron 156 individuos con anticuerpos anti- $T$. cruzi detectados por ELISA y MABA, para una prevalencia general de $7,2 \%$, de los cuales 82 $(52,6 \%)$ eran del sexo masculino y $74(47,4 \%)$ del femenino. El seropositivo de menor edad fue de 9 años, seguido de 7 individuos que tenían entre 10 y 20 años. La seroprevalencia incrementa de $2,3 \%$ a $8,4 \%$ entre los 10 y los 40 años de edad (Figura 1). Sin embargo, la prevalencia aumenta drásticamente para las edades comprendidas entre los 50 hasta los 80 años con valores de $40 \%$ aproximadamente, no obstante la máxima prevalencia se observó a los 90 años, ya que de 6 individuos analizados 4 resultaron seropositivos (Figura 1).

De los 333 cánidos muestreados, se encontraron 23 positivos para anticuerpos anti-T. cruzi detectados con ELISA y MABA, para una prevalencia general de 6,9\%, ubicándose el mayor nú- mero de seropositivos entre los rangos de edades de 2 a 6 años, no hubo casos seropositivos en cánidos mayores de 10 años y en menores de 1 año de edad.

\section{Índices vectoriales}

En 21 de las 26 comunidades exploradas se lograron capturar un total de 235 triatominos, de los cuales $212(90,2 \%)$ pertenecían a la especie $T$. maculata, capturados predominantemente en el peridomicilio, mientras que $23(9,8 \%)$ correspondió a $P$. geniculatus, capturados predominantemente en el domicilio.

De un total de 905 viviendas encuestadas, sólo en 53 se encontraron triatominos; en 10 viviendas se recolectaron ninfas, y en 1 vivienda cohabitaban dos especies de triatominos. De 23 P. geniculatus capturados sólo uno presentó formas parasitarias flagelares similares a T. cruzi en las deyecciones, mientras que para T. maculata no se logró demostrar la presencia del parásito. La Tabla 1 muestra los índices vectoriales calculados a partir de los datos recolectados.

\section{Análisis de los factores de riesgo asociados} a la seropositividad

En primer lugar, al analizar la edad encontramos que estuvo fuertemente asociada a la seropositividad (OR: 1,06; IC95\%: 1,05-1,07; p < 0,0001).

Tabla 1

Índices entomológicos. Parroquia San Miguel, Municipio Urdaneta, Estado Lara, Venezuela, enero 2005-diciembre 2008.

\begin{tabular}{|c|c|c|c|}
\hline Parámetro & $\begin{array}{l}\text { Población de referencia } \\
\text { total }\end{array}$ & Población afectada & Índice (\%) \\
\hline Índice de infestación & 905 * & 53 ** & 5,9 \\
\hline Índice de colonización & $53 * \star$ & $10 * \star \star$ & 18,9 \\
\hline Índice de coinfestación & 53 ** & $1 \#$ & 1,9 \\
\hline Índice de infección del vector & $235 \# \#$ & 1 \#\#\# & 0,004 \\
\hline Índice de infección domiciliaria & $53 * \star$ & $1 \S$ & 1,9 \\
\hline Índice de dispersión & $26 \S \S$ & $21 \S \S \S$ & 80,8 \\
\hline
\end{tabular}

Los superíndices significan:

* Número de viviendas encuestadas;

** Número de viviendas con vectores domiciliarios;

*** Número de viviendas con ninfas y/o huevos domiciliarios:

\# Número de viviendas con más de una especie de triatomino domiciliario;

\#\# Vectores capturados;

\#\#\# Número de vectores positivos a T. cruzi;

$\S$ Número de viviendas con triatominos infectados con T. cruzi;

$\S \S$ Número de sectores estudiados;

$\S \S \S$ Número de sectores donde se capturaron vectores. 
Factores de riesgo relacionados con la forma de transmisión, hábitos biológicos, antecedentes personales y grado de instrucción. Parroquia San Miguel, Municipio Urdaneta, Estado Lara, Venezuela, enero 2005-diciembre 2008.

\begin{tabular}{|c|c|c|c|c|c|c|c|}
\hline \multirow[t]{2}{*}{ Clase/Factor } & \multicolumn{2}{|c|}{ Población total } & \multicolumn{2}{|c|}{ Seronegativos (SN) } & \multicolumn{2}{|c|}{ Seropositivos (SP) } & \multirow[t]{2}{*}{ SP/SN } \\
\hline & $\mathbf{n}$ & $\%$ & $\mathbf{n}$ & $\%$ & $\mathbf{n}$ & $\%$ & \\
\hline \multicolumn{8}{|l|}{ Forma de transmisión } \\
\hline Vivienda anterior con vectores & 490 & 87,8 & 339 & 57,7 & 151 & 61,9 & 1,07 \\
\hline Conocimiento del vector & 444 & 79,6 & 315 & 80,6 & 129 & 77,3 & 0,96 \\
\hline Carnes silvestres & 436 & 78,1 & 305 & 78,0 & 131 & 78,4 & 1,01 \\
\hline Madre con Chagas & 100 & 27,1 & 60 & 23,5 & 40 & 35,1 & 1,49 \\
\hline Contacto con el vector & 123 & 26,5 & 81 & 51,6 & 42 & 52,5 & 1,02 \\
\hline Transfusiones & 71 & 12,7 & 55 & 14,0 & 16 & 9,6 & 0,68 \\
\hline \multicolumn{8}{|l|}{ Hábitos biológicos } \\
\hline Alcohol & 140 & 25,3 & 105 & 27,1 & 35 & 21,0 & 0,77 \\
\hline Chimó & 101 & 22,1 & 59 & 17,8 & 42 & 33,6 & 1,89 \\
\hline Cigarrillo & 61 & 10,9 & 51 & 13,0 & 10 & 6,0 & 0,46 \\
\hline \multicolumn{8}{|l|}{ Antecedentes personales patológicos } \\
\hline Helmintiasis & 263 & 47,1 & 184 & 47,1 & 79 & 47,3 & 1,01 \\
\hline Hipertensión arterial & 194 & 34,8 & 144 & 36,9 & 50 & 29,9 & 0,81 \\
\hline Historia de cáncer familiar & 116 & 20,8 & 92 & 23,5 & 24 & 14,4 & 0,61 \\
\hline Alergias & 114 & 20,5 & 86 & 22,1 & 28 & 16,8 & 0,76 \\
\hline Infecciones bacterianas severas & 63 & 11,3 & 43 & 11,0 & 20 & 12,0 & 1,09 \\
\hline Autoinmunes & 52 & 9,3 & 38 & 9,7 & 14 & 8,4 & 0,86 \\
\hline \multicolumn{8}{|l|}{ Instrucción } \\
\hline Primaria incompleta & 289 & 52,9 & 201 & 52,2 & 88 & 54,7 & 1,05 \\
\hline
\end{tabular}

Nota: conocimiento del vector: el individuo refiere conocer el vector y lo reconoce a partir de un muestrario de insectos; contacto con el vector: el individuo conoce al vector y refiere haber tenido contacto lesivo; carnes silvestres: el individuo refiere haber comido carne de animales silvestres. Los hábitos biológicos no se refieren a conductas adictivas.

Al analizar la relación entre la seropositividad humana individual y las formas de transmisión se obtuvo significancia estadística para "madre con antecedentes de enfermedad de Chagas" (Tablas 2 y 3). Aún cuando el conocimiento de los vectores a partir de un muestrario no estuvieron asociados a la seropositividad (Tabla 2), al discernir el conocimiento por especie, obtuvimos que el "conocimiento de $R$. prolixus" estuvo asociado a la seropositividad (OR: 1,69; IC95\%: 1,09-2,63; $\mathrm{p} \leq$ 0,019).

Para los antecedentes personales y hábitos biológicos observamos una relación significativa como factor de riesgo y seropositividad, el consumo de chimó; mientras que el tabaquismo, consumo de alcohol y antecedentes familiares o personales relacionados con cáncer resultaron factores protectores (Tablas 2 y 3 ).

$\mathrm{Al}$ analizar la relación entre individuos seropositivos, sus viviendas y la referencia de mamíferos reservorios en ellas, observamos, que la presencia de perros, caprinos y armadillos (permanente o temporal de estos animales en la vivienda) son factores de riesgo. También las aves (gallinas y los pájaros) son factores de riesgo (Tablas 2 y 3 ).

Además, se encontró que el orden y distribución del domicilio estuvo asociado a la seropositividad como factores de riesgo (artefactos viejos y/o dañados acumulados, materiales de construcción, limpieza inadecuada y basura libre en el domicilio). Las variables analizadas en el peridomicilio en relación a la distribución y el orden no arrojaron relaciones significativas $(\mathrm{p}>$ $0,05)$, sin embargo, al analizar la correspondencia entre las construcciones anexas o hábitats en el peridomicilio y seropositividad humana en la vivienda, observamos significancia estadística $(\mathrm{p}<0,05)$ para anexos de bajareque, nidos $\mathrm{y}$ cuevas como factores de riesgo, mientras que los depósitos de herramientas trabajo y utensilios del hogar fuera de la casa resultaron ser un factor protector (Tablas 3 y 4 ). 
Factores epidemiológicos asociados a la infección por Trypanosoma cruzi. Parroquia San Miguel, Municipio Urdaneta, Estado Lara, Venezuela, enero 2005-diciembre 2008.

\begin{tabular}{|c|c|c|c|c|c|c|c|}
\hline Factor de riesgo & B & S.E. & Wald & Df & $\mathbf{P}$ & OR & IC95\% \\
\hline Madre con Chagas & 0,55 & 0,23 & 5,81 & 1 & 0,016 & 1,74 & $1,11-2,72$ \\
\hline Cigarrillo & $-0,85$ & 0,37 & 5,35 & 1 & 0,021 & 0,43 & $0,21-0,88$ \\
\hline Chimó & 0,85 & 0,25 & 11,93 & 1 & 0,001 & 2,35 & $1,45-3,81$ \\
\hline Alcohol & $-0,48$ & 0,24 & 4,25 & 1 & 0,039 & 0,62 & $0,39-0,98$ \\
\hline Cáncer & $-0,54$ & 0,25 & 4,67 & 1 & 0,031 & 0,58 & $0,36-0,95$ \\
\hline Perro & 0,68 & 0,22 & 9,54 & 1 & 0,002 & 1,98 & $1,28-3,02$ \\
\hline Caprino & 1,06 & 0,29 & 13,13 & 1 & 0,000 & 2,89 & $1,63-5,13$ \\
\hline Armadillos & 0,87 & 0,23 & 14,73 & 1 & 0,000 & 2,38 & $1,53-3,70$ \\
\hline Gallinas & 1,68 & 0,23 & 55,56 & 1 & 0,000 & 5,35 & $3,44-8,31$ \\
\hline Pájaros & 0,70 & 0,29 & 5,87 & 1 & 0,015 & 2,01 & $1,14-3,52$ \\
\hline Depósitos de herramientas * & $-0,54$ & 0,22 & 6,33 & 1 & 0,012 & 0,58 & $0,38-0,89$ \\
\hline Anexos de bajareque * & 0,63 & 0,22 & 8,58 & 1 & 0,003 & 1,88 & $1,23-2,86$ \\
\hline Cueva de animales * & 0,91 & 0,25 & 13,52 & 1 & 0,000 & 2,48 & $1,53-4,04$ \\
\hline Nidos de pájaros * & 1,08 & 0,31 & 12,27 & 1 & 0,000 & 2,93 & $1,61-5,36$ \\
\hline Artefactos viejos y/o desechos ** & 0,49 & 0,22 & 4,74 & 1 & 0,029 & 1,63 & $1,05-2,52$ \\
\hline Materiales de construcción ** & 1,61 & 0,34 & 22,74 & 1 & 0,000 & 4,99 & $2,58-9,66$ \\
\hline Limpieza inadecuada ** & 1,16 & 0,32 & 13,16 & 1 & 0,000 & 3,20 & $1,71-5,99$ \\
\hline Basura ** & 1,41 & 0,32 & 19,14 & 1 & 0,000 & 4,08 & $2,17-7,66$ \\
\hline
\end{tabular}

* Peridomicilio;

** Domicilio.

\section{Discusión}

En el presente trabajo presentamos datos que permiten asociar factores de riesgo epidemiológicos con la probabilidad de presentar anticuerpos anti-T. cruzi, en poblaciones rurales situadas en un área endémica para la enfermedad de Chagas, actualmente infestada predominantemente por T. maculata.

La seropositividad estuvo fuertemente asociada con la edad, estando la mayor parte de los seropositivos en edades superiores a los 50 años, mientras que no observamos seropositivos en menores a 9 años. Estos resultados plantean que no existe actualmente transmisión activa de la enfermedad en los humanos y que la mayoría de los casos seropositivos, reflejan un problema remoto situado a más de cuatro décadas en el tiempo, lo cual coincide efectivamente con la implementación en Venezuela en el año 1966 de un programa de control de vectores a gran escala, que consistió en la aplicación de insecticidas (Dieldrin) y la mejoría o substitución de ranchos por viviendas; diez años más tarde, en 1976, la infestación domiciliaria descendió de un $31,1 \%$ a 5,6\% (revisado por Briceño-León 13). Posterior a esta intervención gubernamental no ha sido aplicado otro programa a gran escala en la zona, sin embargo, explica la caída de la prevalencia en los individuos nacidos posterior a 1966 (menores a 40 años).

La misma conclusión no parece ser aplicable a los caninos, ya que, observamos una prevalencia del 6,9\% en individuos menores a 10 años de edad, indicando que existe transmisión de la enfermedad en ambientes no domiciliarios. Los valores de prevalencia en caninos observados en este trabajo confirman los datos obtenidos por Rojas et al. 9 en la parroquia de Xaguas (municipio Urdaneta), quienes reportaron una prevalencia del 6,4\%. Cuando la incidencia en humanos es baja, el perro por sus hábitos biológicos y conductuales actúa como indicador o centinela de la existencia de transmisión activa de la enfermedad en las áreas controladas 14 .

En este trabajo se obtuvo una asociación estadísticamente significativa entre el hecho de conocer el vector $R$. prolixus y la seropositividad. $\mathrm{Al}$ analizar este hallazgo en el contexto histórico, parece factible que esta especie colonizaba las viviendas antes de la implementación del programa en 1966, está afirmación es sustentada por Cova-García \& Suárez 15, quienes reportaron 
Factores de riesgo relacionados con los reservorios, las fuentes alimentarias de los vectores e higiene de la vivienda. Parroquia San Miguel, Municipio Urdaneta, Estado Lara, Venezuela, enero 2005-diciembre 2008.

\begin{tabular}{|c|c|c|c|c|c|c|c|}
\hline \multirow[t]{2}{*}{ Clase/Factor } & \multicolumn{2}{|c|}{ Población total } & \multicolumn{2}{|c|}{ Seronegativos (SN) } & \multicolumn{2}{|c|}{ Seropositivos (SP) } & \multirow[t]{2}{*}{$\mathrm{SP} / \mathrm{SN}$} \\
\hline & $\mathrm{n}$ & $\%$ & $\mathrm{n}$ & $\%$ & $\mathrm{n}$ & $\%$ & \\
\hline \multicolumn{8}{|l|}{ Mamíferos domésticos } \\
\hline Perro & 473 & 52,3 & 384 & 49,3 & 89 & 71,2 & 1,44 \\
\hline Gato & 160 & 18,7 & 115 & 15,8 & 45 & 36,0 & 2,28 \\
\hline Porcinos & 94 & 10,4 & 72 & 9,2 & 22 & 17,5 & 1,89 \\
\hline Caprinos & 65 & 7,9 & 41 & 5,3 & 24 & 19,1 & 3,62 \\
\hline Ovinos & 57 & 6,3 & 42 & 5,4 & 15 & 12,0 & 2,23 \\
\hline Bovinos & 39 & 4,3 & 27 & 3,5 & 12 & 9,5 & 2,75 \\
\hline \multicolumn{8}{|l|}{ Mamíferos silvestres } \\
\hline Ratas y ratones & 404 & 44,6 & 326 & 41,9 & 78 & 61,9 & 1,48 \\
\hline Murciélagos & 402 & 44,4 & 332 & 42,6 & 70 & 55,6 & 1,30 \\
\hline Zarigüeyas & 311 & 34,4 & 255 & 32,7 & 56 & 44,4 & 1,36 \\
\hline Armadillos & 154 & 17,0 & 111 & 14,3 & 43 & 34,1 & 2,40 \\
\hline Perezosos & 76 & 8,4 & 53 & 6,8 & 23 & 18,4 & 2,70 \\
\hline Osos hormigueros & 46 & 5,1 & 29 & 3,7 & 17 & 13,6 & 3,65 \\
\hline \multicolumn{8}{|l|}{ Aves } \\
\hline Gallinas & 338 & 37,4 & 245 & 31,5 & 93 & 73,8 & 2,35 \\
\hline Pájaros & 69 & 7,6 & 44 & 5,7 & 25 & 19,8 & 3,51 \\
\hline Palomas & 51 & 5,6 & 38 & 4,9 & 13 & 10,3 & 2,12 \\
\hline Pavos & 51 & 5,6 & 36 & 4,6 & 15 & 11,5 & 2,50 \\
\hline Gansos & 6 & 0,7 & 4 & 0,5 & 2 & 1,6 & 3,09 \\
\hline \multicolumn{8}{|l|}{ Domicilio } \\
\hline Rancho & 131 & 46,5 & 55 & 31,6 & 76 & 70,4 & 2,23 \\
\hline Desorden 1 & 257 & 43,2 & 180 & 37,7 & 77 & 65,8 & 1,75 \\
\hline Desorden 2 & 193 & 32,4 & 134 & 28,0 & 59 & 50,4 & 1,80 \\
\hline Desorden 3 & 181 & 30,4 & 126 & 26,3 & 55 & 47,4 & 1,80 \\
\hline Desorden 4 & 101 & 17,0 & 70 & 14,6 & 31 & 26,5 & 1,81 \\
\hline Desorden 5 & 73 & 12,2 & 51 & 10,7 & 22 & 18,8 & 1,77 \\
\hline \multicolumn{8}{|l|}{ Peridomicilio } \\
\hline Gallineros & 322 & 35,6 & 278 & 35,7 & 44 & 34,9 & 0,98 \\
\hline Anexos de bajareque & 301 & 33,3 & 242 & 31,1 & 59 & 46,8 & 1,51 \\
\hline Cueva de animales & 106 & 11,7 & 75 & 9,6 & 31 & 24,6 & 2,56 \\
\hline Depósito de herramientas & 69 & 7,6 & 44 & 5,7 & 25 & 19,8 & 3,51 \\
\hline Nidos de pájaros & 59 & 6,5 & 39 & 5,0 & 20 & 15,9 & 3,17 \\
\hline Palomares & 6 & 0,7 & 4 & 0,5 & 2 & 1,6 & 3,09 \\
\hline Desorden PD 1 & 206 & 53,9 & 146 & 53,5 & 60 & 55,1 & 1,03 \\
\hline Desorden PD 2 & 184 & 48,3 & 128 & 47,1 & 56 & 51,4 & 1,09 \\
\hline Desorden PD 3 & 196 & 51,6 & 136 & 50,0 & 60 & 55,6 & 1,11 \\
\hline Desorden PD 4 & 170 & 44,6 & 122 & 44,9 & 48 & 44,0 & 0,98 \\
\hline Desorden PD 5 & 147 & 38,6 & 99 & 36,4 & 48 & 44,0 & 1,21 \\
\hline Desorden PD 6 & 103 & 27,0 & 73 & 26,8 & 30 & 27,5 & 1,03 \\
\hline
\end{tabular}

Desorden 1: mobiliario y/o ropa mal arreglados; desorden 2: artefactos viejos o dañados amontonados; desorden 3: limpieza inadecuada; desorden 4: basura fuera de contenedores; desorden 5: materiales de construcción amontonados; desorden PD 1: botellas, latas, recipientes vacíos y cauchos desparramados; desorden PD 2: limpieza inadecuada; desorden PD3: restos de: vidrio, plástico o metal desparramados; desorden PD4: basura amontonada fuera de los contenedores; desorden PD5: artefactos viejos y/o dañados desparramados; desorden PD6: escombros y/o materiales de construcción amontonados. 
la presencia de $R$. prolixus y $T$. maculata en la zona para la década del 1950 a 1960.

La especie T. maculata, posiblemente se encuentra en la actualidad en un proceso de cambios de las frecuencias de formas adaptadas a determinados ambientes. Tradicionalmente esta especie ha sido reconocida como ornitófaga y más adaptada a los ambientes peridomésticos (gallineros), debido a su frecuente presencia en estos ecótopos 16. Estudios recientes sobre la estructura espacial de $T$. maculata de la localidad de Cauderales 17, en el mismo municipio del presente estudio, se encontró presencia de adultos tanto en el domicilio como en el peridomicilio y que la presencia domiciliaria obedecía a reinfestaciones desde el peridomicilio, es decir, no se detectaron formas distintas entre los triatominos de estos dos ambientes, no estando así esta especie estructurada espacialmente en esta localidad. Sin embargo, la capacidad vectorial de T. maculata es limitada, como consecuencia de sus bajos índices de infección y la reducida viabilidad cuando se alimenta con sangre humana 18 .

La asociación entre los antecedentes maternos de Chagas y la seropositividad, podría involucrar a la trasmisión transplacentaria como responsable de la presencia de anticuerpos anti-T. cruzi. En áreas donde no existe transmisión vectorial, ha sido demostrado que la transmisión transplacentaria puede ocurrir hasta tres generaciones 19 , no obstante, en áreas endémicas los antecedentes maternos podrían simplemente reflejar la situación epidemiológica de la familia, la cual vive en un entorno de pobreza con presencia de factores de riesgo para la infección por T. cruzi.

La relación entre el consumo de chimó y el habitó de fumar con la probabilidad de seropositividad, involucra aspectos culturales, inmunológicos y farmacológicos. El chimó es una pasta preparada a partir de hojas de tabaco y se consume mediante masticación. Los aspectos culturales del chimó se traducen en una costumbre ancestral amerindia prehispánica, que fue asumida en la modernidad por las poblaciones obreras de las zonas agropecuarias, motivada por las propiedades farmacológicas y terapéuticas de la nicotina; la cual mitiga el deseo de comer y activa el sistema nervioso central, permitiendo al obrero un mayor rendimiento físico y económico en su jornada laboral 20.

Recientemente ha sido reportado que la nicotina es capaz de promover la entrada de $\mathrm{Ca}^{2+}$ al citoplasma del T. cruzi 21. Este evento está asociado con la proliferación, diferenciación e invasión parasitaria, lo que podría garantizar un contacto efectivo con las células involucradas en la respuesta inmune 22 , explicando una mayor probabilidad de seropositividad en individuos consumidores de chimó que hayan sido infectados por T. cruzi. Sin embargo, al plantear el hecho que parte de los individuos afectados adquirieron la infección durante la edad lactante y pre-escolar, implica que el hábito de consumir chimó se inicia en etapas tempranas de la edad escolar, en este sentido ha sido reportado en Venezuela que la edad promedio de iniciación en el hábito es 11,6 años 23. De esta manera, la nicotina mantendría una respuesta inmune robusta en el tiempo, disminuyendo la tasa de seronegativización espontánea en los individuos consumidores de chimó 24 .

El hecho de que el chimó sea consumido por la población obrera rural, podría asociar la seropositividad con factores socioeconómicos relacionados con la pobreza extrema. Con anterioridad, en las zonas rurales de Venezuela ha sido reportado asociación entre electrocardiogramas anormales con el consumo de chimó 25 . La asociación protectora con el hábito de fumar podría explicarse porque el humo es repelente eficiente de los insectos 26 .

El consumo de alcohol estuvo asociado negativamente con la seropositividad, similares resultados han sido descritos en las zonas rurales de Venezuela para la relación entre anormalidades en el electrocardiograma y el consumo de alcohol 25. El consumo habitual del alcohol afecta la inmunidad celular especialmente la relacionada con la respuesta inmune específica contra antígenos, ya que, disminuye la función de las células presentadoras de antígenos 27 y disminuye la producción de linfocitos B 28 , estos fenómenos explicarían la baja probabilidad de respuesta humoral en individuos que hayan tenido contacto con el T. cruzi.

La relación entre cáncer y enfermedad de Chagas recibió atención con los estudios pioneros de Roskin y colaboradores a partir de 1930 (referencias en Kremenstsov 29 ) y recientemente hay renovado interés en el estudio de las propiedades antineoplásicas del T. cruzi 30. En este trabajo los resultados demuestran que hubo una relación negativa entre antecedentes personales y familiares de cáncer con la probabilidad de seropositividad. En el contexto sociocultural y epidemiológico, podría ser lógico sugerir que los familiares de los casos seropositivos han vivido en entornos con factores de riesgo para ser infectados con $T$. cruzi y por lo tanto tienen más probabilidades de ser seropositivos que los familiares de los pacientes seronegativos. Esto explicaría que los pacientes seropositivos tengan una menor probabilidad de padecer cáncer, como consecuencia de la actividad antineoplásica directa de carácter invasiva o indirecta de carác- 
ter inmunogénico de $T$. cruzi 29,30 . No obstante, la frecuencia de la asociación positiva entre las formas digestivas de la enfermedad de Chagas y las neoplasias malignas digestivas, han sido reportadas que oscilan entre un 3,4 y un 9,2\%, especialmente las esofagopatías 31 . En Venezuela no han sido descritas formas digestivas de la enfermedad de Chagas.

Estudios previos realizados en otras regiones de Latinoamérica han identificado características del domicilio y del peridomicilio asociadas a la seropositividad. Por ejemplo, las casas con paredes de adobe y caña o con techos de palma, así como la presencia de leña en el peridomicilio fueron asociadas con un mayor riesgo de seropositividad; mientras que la acumulación de basura, materia orgánica, piedras y madera, el número de corrales de animales en el peridomicilio estuvieron asociados negativamente con la seropositividad 32,33. En este trabajo observamos una asociación positiva entre el acúmulo de artefactos viejos, desechos, materiales de construcción, basura y la limpieza inadecuada del domicilio con la probabilidad de los habitantes de la vivienda de ser seropositivos. Esto refleja una cultura relacionada con la pobreza arrastrada a lo largo de los años, que ha permitido la supervivencia de los vectores en los ambientes domiciliarios, al ofrecerles lugares de refugios, poco movilizados y con fuentes inmediatas de alimento, que les permite colonizar la vivienda por largo tiempo, aumentando la probabilidad de la transmisión vectorial intradomiciliaria de la enfermedad de Chagas.

La presencia de anexos de bajareque, cuevas de animales y nidos de pájaros en el peridomicilio se asociaron efectivamente con la seropositividad, mientras que la existencia de depósitos para herramientas de trabajo se asoció negativamente. Los anexos de bajareque representan hábitats donde los triatominos consiguen refugio y fuentes de alimentación, permitiendo su proliferación y supervivencia; la existencia de nidos y cuevas son reflejo de casas ubicadas en ambientes selváticos. La asociación negativa observada para el depósito de herramientas puede ser interpretada como un mejoramiento de las condiciones de vida y una mejor actitud hacia la higiene doméstica 33.

En este trabajo observamos una relación positiva entre la presencia de perros, caprinos o gallinas en el domicilio con la seropositividad en humanos. En trabajos previos, la presencia de perros y gallinas en el área domiciliaria han sido asociados con la seropositividad en humanos 33 . Los perros representan reservorios para la infección por T. cruzi, la presencia de perros infectados en el domicilio está fuertemente correlacionada con un mayor número de triatominos infectados y una mayor prevalencia de enfermedad de Chagas en niños 33,34 . Sin embargo, en este trabajo no hubo correlación entre las casas con individuos seropositivos y las casas con perros seropositivos, por lo tanto, el perro actualmente no se está comportando como un reservorio, sino que estaría marcando una costumbre de las familias seropositivas en relación con la preferencia o necesidad de convivir con estos animales. Las gallinas son fuentes de alimento para los triatominos y su presencia en las aéreas domiciliarias impactan la ecología doméstica de la enfermedad de Chagas, ya que, incrementan la infestación del domicilio, conllevando a un incremento en el contacto del vector con los reservorios domésticos, aumentando de esta manera el número de triatominos infectados 35,36. En este sentido ha sido sugerido que el éxito reproductivo de $R$. prolixus se ve favorecido con mayor fecundidad si en el domicilio ocurriera la ingesta con sangre humana, pero si previamente el insecto se ha alimentado sobre gallina 37 .

La asociación entre seropositividad y la presencia de pájaros y armadillos podría estar relacionada con viviendas localizadas en ambientes selváticos. Los pájaros como fuente de alimento y los armadillos como reservorio, son animales que logran mantener los ciclos peridoméstico y selvático de la enfermedad de Chagas 38 .

En conclusión, la transmisión de la enfermedad de Chagas a humanos en la parroquia San Miguel es un fenómeno remoto asociado a $R$. prolixus, relacionado a costumbres relacionadas con la pobreza y con la construcción de viviendas en las áreas silvestres. Sin embargo, aspectos relacionados con los hábitos del huésped pueden impactar la respuesta inmune producto de la infección parasitaria y podrían determinar el curso de la enfermedad.

Los aspectos más importantes de este trabajo es haber demostrado que la transmisión de la enfermedad de Chagas en la parroquia San Miguel está actualmente interrumpida en los humanos y haber delimitado los factores de riesgo asociados a la infección. Dado que los factores de riesgo para la transmisión persisten en la región estudiada, los resultados permiten sugerir el establecimiento de medidas educativas dirigidas a las familias a adquirir una cultura cognoscitiva que les permita reconocer al vector, sus fuentes de alimento y hábitats; conocer los modos de transmisión y los reservorios. Adquirir una cultura de higiene respecto a la limpieza, el orden, la disposición de los enseres domésticos y de la basura; a la adecuada distribución espacial de las construcciones anexas respecto al domicilio; a la adecuada situación espacial e higiénica de los 
lugares de alojamiento de los animales domésticos; a la eliminación de las cuevas y nidos de animales silvestre en el peridomicilio y al fomento de hábitos sanos en el individuo y la familia. Los entes del estado deben establecer una vigilancia sobre los índices de infestación e infección de los vectores y sobre la incidencia de la enfermedad en la población canina.

La limitación principal de este estudio deriva de que la enfermedad de Chagas en humanos en la región está interrumpida actualmente y que la prevalencia de la infección estuvo significativamente asociada con edades avanzadas, esto con- lleva a concluir que los factores de riesgo obtenidos reflejan una situación remota y no refleja la dinámica de la transmisión en la actualidad, por lo tanto los datos no son necesariamente aplicables a poblaciones donde actualmente exista transmisión activa de la enfermedad.

Estudios futuros deberían estar dirigidos a establecer los modos de transmisión y los factores epidemiológicos asociados a la transmisión de la enfermedad de Chagas en los caninos, determinar como la nicotina afecta el curso de la infección por T cruzi e incentivar la investigación sobre las propiedades antineoplásicas del parásito.

\section{Resumen}

Determinamos factores de riesgo asociados a la seropositividad para anticuerpos anti-Trypanosoma cruzi en 26 poblaciones rurales, 905 viviendas, 2.156 individuos y 333 caninos en el Estado Lara, Venezuela. La seropositividad fue determinada mediante ELISA y MABA. Los datos fueron obtenidos mediante encuestas entomológicas, demográficas y médicas. Los factores de riesgo fueron establecidos mediante regresión logística binaria. La seroprevalencia humana fue de 7,24\% y la canina 6,9\%. La seropositividad estuvo asociada positivamente al Rhodnius prolixus, la edad, madre con antecedentes de Chagas, consumo de chimó, presencia de mamíferos y aves en la vivienda, desorden en el domicilio, y anexos de bajareque, nidos y cuevas en el peridomicilio. Negativamente con hábitos de consumo de tabaco y alcohol, antecedentes de cáncer y a depósitos en el peridomicilio. En conclusión, la enfermedad de Chagas en el área rural estudiada es un fenómeno remoto transmitida por $R$. prolixus y vía transplacentaria, asociada a hábitos socioculturales relacionados con la pobreza, a entornos selváticos y antecedentes médicos del huésped.

Enfermedad de Chagas; Factores de Riesgo; Serología

\section{Colaboradores}

R. Bonfante-Cabarcas y C. Rodríguez-Bonfante contribuyeron con la coordinación general del proyecto, trabajo de campo, análisis de los datos y redacción del manuscrito. B. O. Vielma contribuyó con el trabajo de campo, análisis e interpretación de los datos, revisión crítica y aprobación final de la versión a ser publicada. D. García contribuyó con el análisis estadístico, revisión crítica y aprobación final de la versión a ser publicada. A. Mogollón-Saldivia contribuyó con el trabajo de campo relacionado con el manejo de animales, análisis de los datos de los caninos, revisión crítica y aprobación final de la versión a ser publicada. E. Aldana contribuyó con la sistemática de triatominos capturados, análisis, redacción y discusión del manuscrito. J. L. C. Curvelo contribuyó con el diseño, montaje e interpretación del inmunodiagnóstico; análisis, redacción y discusión del manuscrito.

\section{Agradecimientos}

Los autores expresan su reconocimiento al trabajo realizado por los alumnos del sexto año de Medicina del Decanato de Ciencias de la Salud de la Universidad Centroccidental Lisandro Alvarado, Barquisimeto, Venezuela. 


\section{Referencias}

1. Armaganijan L, Morillo CA. Chagas disease: 101 years of solitude! Time for action. Stroke 2010; 41:2453-4.

2. Chagas disease (American trypanosomiasis) fact sheet (revised in June 2010). Wkly Epidemiol Rec 2010; 85:334-6.

3. World Health Organization. Chagas disease: control and elimination. Report of the Secretariat. http://apps.who.int/gb/ebwha/pdf_files/EB124/ B124_17-en.pdf (accedido el 21/May/2011).

4. Organización Panamericana de la Salud. Estimación cuantitativa de la enfermedad de Chagas en las Américas. Montevideo: Organización Panamericana de la Salud; 2006. http://www.bvsops.org. uy/pdf/chagas19.pdf (accedido el 21/May/2011).

5. Aché A, Matos AJ. Interrupting Chagas' disease transmisión in Venezuela. Rev Inst Med Trop São Paulo 2001; 43:37-43.

6. Feliciangeli D, Campbell-Lendrum D, Martínez C, González D, Coleman P, Davies C. Chagas disease control in Venezuela: lessons for the Andean region and beyond. Trends Parasitol 2003; 19:44-9.

7. Alarcón de Noya B, Díaz-Bello Z, Colmenares C, Ruiz-Guevara R, Mauriello L, Zavala-Jaspe R, et al. Large urban outbreak of orally acquired acute Chagas disease at a school in Caracas, Venezuela. J Infect Dis 2010; 201:1308-15.

8. Feliciangeli MD, Sanchez-Martin MJ, Suarez B, Marrero R, Torrellas A, Bravo A, et al. Risk factors for Trypanosoma cruzi human infection in Barinas state, Venezuela. Am J Trop Med Hyg 2007; 76:915-21.

9. Rojas ME, Várquez P, Villarreal MF, Velandia C, Vergara L, Morán-Borges YH, et al. Estudio seroepidemiológico y entomológico sobre la enfermedad de Chagas en un área infestada por Triatoma maculata (Erichson 1848) en el centro-occidente de Venezuela. Cad Saúde Pública 2008; 24:2323-33.

10. Añez N, Crisante G, Rojas A, Díaz N, Añez-Rojas N, Carrasco $\mathrm{H}$, et al. La cara oculta de la enfermedad de Chagas de Venezuela. Bol Malariol Salud Ambient 2003; 43:45-57.

11. Lent H, Wygodzinsky P. Revision of the Triatominae (Hemiptera, Reduviidae) and their significance as vectors of Chagas' disease. Bull Am Mus Nat Hist 1979; 163:123-520.

12. Silveira AC, Sanches O. Guía para muestreo de actividades de vigilancia y control vectorial de la enfermedad de Chagas. http://www.bvsops.org.uy/ pdf/chagas09.pdf (accedido el 21/May/2011).

13. Briceño-León R. La enfermedad de Chagas en las Américas: una perspectiva de ecosalud. Cad Saúde Pública 2009; 25 Suppl 1:S71-82.

14. Castañera MB, Lauricella MA, Chuit R, Gurtler RE. Evaluation of dogs as sentinels of the transmission of Trypanosoma cruzi in a rural area of northwestern Argentina. Ann Trop Med Parasitol 1998; 92:671-83.

15. Cova-García P, Suárez MA. Estudio de los triatominos en Venezuela. Caracas: Tipografía Vargas S.A.; 1959. (Publicaciones de la División de Malariología, 11).
16. Tonn R, Otero M, Mora E, Espinola H, Carcavallo R. Aspectos biológicos, ecológicos y distribución geográfica de Triatoma maculata (Erichson, 1848), (Hemiptera, Reduviidae) en Venezuela. Bol Dir Malariol Saneam Ambient 1978; 18:16-24.

17. Soto Vivas A, Rodríguez C, Bonfante-Cabarcas R, Aldana E. Morfometría geométrica de Triatoma maculata (Erichson, 1848) de ambientes doméstico y peridoméstico, estado Lara, Venezuela. Bol Malariol Salud Ambient 2007; 47:231-5.

18. Torres K, Avendaño F, Lizano E, Rojas M, Rodríguez-Bonfante C, Bonfante-Cabarcas R, et al. Evaluación de la estructura espacial de Triatoma maculata del centrooccidente de Venezuela y su viabilidad alimentando con sangre humana en condiciones de laboratorio Triatoma maculata de Venezuela. Biomédica 2010; 30:72-81.

19. Mallimaci MC, Sijvarger C, Dates A, Álverez M, Sosa-Estani S. Seroprevalencia de la enfermedad de Chagas en Ushuaia, Argentina, una zona sin triatominos. Rev Panam Salud Pública 2001; 9:169-71.

20. Wilbert J. El significado cultural del uso del tabaco en Sudamérica. http://cpu2001.awardspace.com/ biblodig/bibloarchiv/wilbert1.pdf (accedido el 21/ May/2011).

21. Bollo M, Venera G, de Jiménez Bonino MB, Machado-Domenech E. Binding of nicotinic ligands to and nicotine-induced calcium signaling in Trypanosoma cruzi. Biochem Biophys Res Commun 2001; 281:300-4.

22. Lammel EM, Barbieri MA, Wilkowsky SE, Bertini F, Isola EL. Trypanosoma cruzi: involvement of intracellular calcium in multiplication and differentiation. Exp Parasitol 1996; 83:240-9.

23. Rivera H, Bentolila R, Santos M, Socorro M, Gómez $\mathrm{D}$, Torres J, et al. Uso del tabaco no fumado (tnf), chimó en niños y adolescentes en el Estado Portuguesa. Acta Odontol Venez 2010; 48:1-10.

24. Marin-Neto JA, Rassi Jr. A, Avezum Jr. A, Mattos AC, Rassi A, Morillo CA, et al. The BENEFIT trial: testing the hypothesis that trypanocidal therapy is beneficial for patients with chronic Chagas heart disease. Mem Inst Oswaldo Cruz 2009; 104 Suppl 1:319-24.

25. Novoa-Montero D. ¿Cardiopatía crónica endémica rural chagásica, o crónica común en chagásicos y no chagásicos?: (Conclusiones de veinte años de investigaciones epidemiológicas y clínico-cardiológicas en Venezuela). Rev Soc Venez Microbiol 2002; 22:188-98.

26. Vernède R, van Meer MM, Alpers MP. Smoke as a form of personal protection against mosquitos, a field study in Papua New Guinea. Southeast Asian J Trop Med Public Health 1994; 25:771-5.

27. Szabo G. Consequences of alcohol consumption on host defence. Alcohol Alcohol 1999; 34:830-41.

28. Schleifer SJ, Keller SE, Czaja S. Major depression and immunity in alcohol-dependent persons. Brain Behav Immun 2006; 20:80-91.

29. Krementsov N. Trypanosoma cruzi, cancer and the Cold War. Hist Ciênc Saúde-Manguinhos 2009; 16 Suppl 1:75-94. 
30. Atayde VD, Jasiulionis MG, Cortez M, Yoshida N. A recombinant protein based on Trypanosoma cruzi surface molecule gp 82 induces apoptotic cell death in melanoma cells. Melanoma Res 2008; 18:172-83.

31. Adad SJ, Etchebehere RM, Araujo JR, Madureira AB, Lima VG, Silva AA, et al. Association of chagasic megacolon and cancer of the colon: case report and review of the literature. Rev Soc Bras Med Trop 2002; 35:63-8.

32. Black C, Ocaña S, Riner D, Costales J, Lascano M, Davila S, et al. Household risk factors for Trypanosoma cruzi seropositivity in two geographic regions of Ecuador. J Parasitol 2007; 93:12-6.

33. Gürtler R, Chuit R, Cécere M, Castañera M, Cohen J, Segura E. Household prevalence of seropositivity for Trypanosoma cruzi in three rural village in northwest Argentina: environmental, demographic and entomologic associations. Am J Trop Med Hyg 1998; 59: 741-9.

34. Gürtler RE, Cécere MC, Rubel DN, Petersen RM, Schweigmann NJ, Lauricella MA, et al. Chagas disease in north-west Argentina: infected dogs as a risk factor for the domestic transmission of Trypanosoma cruzi. Trans R Soc Trop Med Hyg 1991; 85:741-5.
35. Cécere M, Gürtler R, Chuit R, Cohen J. Effects of chicken on the prevalence of infestations and population density of Triatoma infestans in rural houses of north-west Argentina. Med Vet Entomol 1997; 11:383-8.

36. Gürtler R, Cohen J, Cécere M, Lauricella M, Chuit $\mathrm{R}$, Segura E. Influence of humans and domestic animals on the household prevalence of Trypanosoma cruzi in Triatoma infestans populations in northwest Argentina. Am J Trop Med Hyg 1998; 58:748-58.

37. Aldana E, Jácome D, Lizano E. Efecto de la alternación de fuentes sanguíneas sobre la fecundidad y la fertilidad de Rhodnius prolixus Stål (Heteroptera: Reduviidae). EntomoBrasilis 2009; 2:17-23.

38. Roque AL, Xavier SC, da Rocha MG, Duarte AC, D'Andrea PS, Jansen AM. Trypanosoma cruzi transmission cycle among wild and domestic mammals in three areas of orally transmitted Chagas disease outbreaks. Am J Trop Med Hyg 2008; 79:742-9.

Recibido el 19/Jul/2010

Versión final presentada el 29/May/2011

Aprobado el 06/Jul/2011 